\title{
Scheduling of multi load AGVs in FMS by modified memetic particle swarm optimization algorithm
}

\author{
V.K. Chawla ${ }^{a^{*}}$, Arindam Kumar Chanda ${ }^{\mathrm{b}}$ and Surjit Angra ${ }^{\mathrm{c}}$
}

${ }^{a}$ Researcher, Mechanical Engineering Department ,NIT Kurukshetra, India

${ }^{b}$ Associate Professor, Department of Mechanical and Automation Engineering, G. B. Pant Engineering College, Okhla, Delhi, India ${ }^{c}$ Professor \& Head in the Department of Mechanical Engineering, National Institute of Technology Kurukshetra, India

\section{CH R O I C L E AB S T R A C T}

Article history:

Received: July 5, 2017

Received in revised format: $\mathrm{Au}$ gust 28, 2017

Accepted: September 27, 2017

Available online:

September 27, 2017

Keywords:

Flexible Manufacturing System

Memetic Algorithm

Modified Memetic Particle Swarm

Optimization

Multi Load AGVS

Particle Swarm Optimization

Scheduling

\begin{abstract}
Use of Automated guided vehicles (AGVs) is highly significant in Flexible Manufacturing System (FMS) in which material handling in form of jobs is performed from one work center to another work center. A multifold increase in through put of FMS can be observed by application of multi load AGVs. In this paper, Particle Swarm Optimization (PSO) integrated with Memetic Algorithm (MA) named as Modified Memetic Particle Swarm Optimization Algorithm (MMPSO) is applied to yield initial feasible solutions for scheduling of multi load AGVs for minimum travel and waiting time in the FMS. The proposed MMPSO algorithm exhibits balanced exploration and exploitation for global search method of standard Particle Swarm Optimization (PSO) algorithm and local search method of Memetic Algorithm (MA) which further results into yield of efficient and effective initial feasible solutions for the multi load AGVs scheduling problem.
\end{abstract}

2018 Growing Science Ltd.

\section{Introduction}

Automated Guided Vehicle systems (AGVs) have been extensively used in flexible manufacturing systems. Efficiency of AGVs and manufacturing facility can be significantly increased by synchronizing various operations for integrated scheduling of manufacturing systems and AGV systems. In FMS jobs with multi machining sequences seek for optimum production sequence and operation start time, while AGVs operating in FMS seek for optimum scheduling (optimum arrival and departure time) along with dispatching and conflict free routing. Mantel and Lander weerd (1995) reported about transportation and production interaction. In general, transportation and production schedules for mass production systems operating with conveyor and forklifts are controlled by pull type policy. FMS employing AGVs has very dynamic schedule and it is highly tedious to know job transfer time with conflict free

* Corresponding author. Tel.: +919312660065

E-mail address: kumar6150046@nitkkr.ac.in (V. K. Chawla) 
route. The generation of optimum schedules of material transfer highly depends on optimum dispatching rule along with conflict free route (Akturk et al., 1996; Veeravalli et al., 2002).

Any FMS consists of various work centers operating in a facility namely CNC milling, assembly, deburring, washing, painting, coating, packaging and material handling equipment (AGVs), etc. AGV operates in the FMS facility to transfer jobs from one pick - up / delivery (P/D) station to another pickup / delivery (P/D) station. The various work centers in FMS are connected with each other by their pick - up / delivery (P/D) stations, which are further connected with each other by a network of guide paths. The AGV transfers jobs in pallets while cruising on network of guide paths from one work center's pick - up / delivery (P/D) station to another work center's pick-up / delivery (P/D) station as shown in Fig. 1. AGV steers automatically to complete its assigned task. AGVs can be further classified as unidirectional, bidirectional, unit load and multi load, etc. Unidirectional AGVs can cruise forward on a guide path in single direction only while bidirectional AGVs are capable to steer in both directions on the same guide path (Egbelu \& Tanchoco, 1986; Gaskins et al., 1989; Gaskins \& Tanchoco, 1987). Unit load AGV can transport single load from one work center to another while multi load AGV system is capable of picking up and deliver more than one load at the same time to any work center which increase through material handling system and FMS facility considerably (Ulusoy et al., 1997; Van Der Meer, 2000). Multi load AGVs have significant potential to increase overall FMS through put and the flexibility. AGVs scheduling, dispatching and conflict free routing to work center can be executed simultaneously or separately. Simultaneous scheduling for work centers and AGVs can lead to increase in throughput of the manufacturing system along with optimum utilization of material handling resources and also reduce earliness and tardiness for the AGVs operating in any manufacturing system. Simultaneous scheduling of work centers and AGVs is more complex however its results are more efficient in comparison with separate scheduling processes of work center and AGVs. The complexity of scheduling and efficiency of system increases more if multi load AGVs are employed in place of unit load AGVs for material handling operations in FMS. From the study of the literature, a potential research gap to schedule multi load $\mathrm{AGVs}$ and jobs processed with minimum travel and waiting time at work centers of FMS is observed. In this paper, an analytical model for simultaneous scheduling of multi load AGVs and jobs in process with minimum travel and waiting time at work centers of FMS is proposed. A new Modified Memetic Particle Swarm Optimization Algorithm (MMPSO) is applied for generation of initial feasible solutions for the aforesaid problem. The solutions generated from MMPSO algorithm was compared with solutions generated by the conventional Particle Swarm Optimization (PSO) algorithm and it was observed that the MMPSO algorithm outperforms PSO algorithm for random and deterministic scheduling conditions.

The information in the paper is represented in several sections .In section 2 literature review on the multi load AGVs is reported. Section 3 includes the problem statement, assumptions, objective function and mathematical formulation of the problem. In Section 4, the metaheuristic algorithms are discussed namely Particle Swarm Optimization (PSO), Memetic Algorithm (MA) and combination of both algorithms named as Modified Memetic Particle Swarm Optimization Algorithm (MMPSO) shown in Fig. 2. Experimental results yielded from the solution of the problem for its initial feasible solutions are discussed in Section 5. The initial feasible solutions and percentage change in the solutions when solved by standard PSO algorithm and by MMPSO algorithm is presented in Table 1 and their analysis by student's t test is represented in Table 2 and Table 3. Section 6 sums up with conclusion and possible future direction of work.

\section{Literature Review}

Scheduling of manufacturing systems and their operations have been investigated by many researchers. Initially many researchers have addressed the work center and unit load AGV scheduling as two independent problems. Later on, researchers focused on integrated scheduling of Machines and unit load 
AGVs. However, only a few researches had been carried out on the significance and potential benefits of scheduling work centers and multi load AGVs functioning in FMS.

The AGV schedule assigns tasks and sends AGVs to Pickup/Drop-of (P/D) jobs. AGVs perform schedule task with certain conditions or by obeying few constraints namely deadlines, priority, etc. A good AGV schedule confirms optimum utilization of resources or minimum processing times for system resources, such as minimizing the number of AGVs used in FMS with no deterioration in system throughput, or confirms minimum total travel time of all AGVs in the FMS (Akturk et al., 1996).

The following listed phenomena occur when scheduling and routing of AGVs are not done carefully as reported by Qiu et al. (2002).

a. Collisions - If more than one AGV attempts to occupy the same path segment at the same time, then there are chances of collision.

b. Congestion - If numbers of AGVs are very high on a guide path segment then situation of congestion arises therefore, for a period of time, there are too many AGVs on a path. Frequent congestion on a path lead to low throughput or deadlock.

c. Live locks - Condition of live locks are generated at intersection. At intersection the AGVs on one path is given higher priority to cruise in comparison to AGVs cruising over other path.

d. Deadlocks - Condition of deadlock arises with multiple AGVs on the same path mutually waiting for release of a job.

It is assumed that the AGVs are reliable and travel at a certain predetermined average speed, so that situations of collisions, breakdowns, and live locks, as well as deadlocks do not occur. The scheduling problem can be divided into two types, static and dynamic. In static scheduling, load transfer requests are predefined. The AGV routes can be analyzed optimized and can be developed before AGV move on to them. In static scheduling AGVs operating in any manufacturing system are scheduled to perform the assigned task. Any change in arrival time of job, change in driving time or any AGV breakdown cause adverse effect on the planning of schedule. In order to obtain optimum utilization of resources, the real time manufacturing operations in FMS should be dynamically scheduled and analyzed. In real time manufacturing conditions the job arrival/departure, time of job loading/unloading and AGV cruising times are not constant and fluctuates during manufacturing operations which makes manufacturing conditions stochastic and highly dynamic in nature hence the situation requires dynamic scheduling and dynamic updating of schedule with time. The dynamic schedule of AGV is updated on the basis of information received for every new job pick/drop assignment.

In order to maximize profit in manufacturing operations the time associated with manufacturing system activities should be minimized which can be achieved by online scheduling. Total minimum time in material handling activities can be obtained in dynamic scheduling by minimizing idle AGV travel time, delay time, time wastage due to rejection of loads etc. Nayyar et al. (1993) analyzed the effect of vivid dispatching rules when applied to multi load AGVs, on the overall efficiency of an AGV system. Authors validated their results by doing comparison between the performance of a fleet comprising single load vehicles and multi-load vehicles. Levitin et al. (2003) formulated AGV problem in which the multi load AGV visits each work center only once according to LIFO rule. Authors also investigated an algorithm to find shortest route among the available routes and validated the performance of algorithm by solving problems available in the literature. Yang et al. (2004) applied time window constraint in real time manufacturing conditions in online scheduling and generated feasible scheduling solutions. It was observed that a time window constraint with generation of new service request also generates a new assignment schedule for AGVs in operation. Meersmans (2002) developed the dynamic schedule for AGVs serving in material handling facility by applying a beam search algorithm heuristic. The scheduling was dependent on the length of planning horizon and after completion of the planning horizon a rescheduling was carried out. Performance of beam search algorithm was found to be better with longer planning horizons and frequent rescheduling. Powell et al. (2000) and Fleischmann et al. (2004) 
also proposed similar research on AGV scheduling. Grunow et al. (2005) investigated a flexible priority rule and MILP based approach for finding and using availability of vehicles. Authors applied flexible priority rule and the MILP approach for different cases considering total lateness of the AGVs. Jerald et al. (2006) applied Adaptive Genetic Algorithm (AGA) to minimize the penalty cost and machine idle time by simultaneous scheduling of AGVs. The results were compared with results of conventional genetic algorithm and it was observed that AGA outperforms conventional genetic algorithm.

Kumar and Sridharan (2010) simulated discrete event models to develop regression based meta models in a Flexible Manufacturing System (FMS). The FMS activities were scheduled using seven scheduling rules on the AGV. They observed that the scheduling algorithm/rules such as Koulamas Algorithm, Fewest Number Of Operations (FNOP), and Earliest Modified Due-Date (EMDD) provide better performance for the various measures such as mean flow time, mean tardiness, and percentage tardy as performance parameters for the job transport operation by the AGV system. They found that flow time as critical indicator for the lead time and flow time can be utilized for setting due date and due date allowances. Rashidi et al. (2010) and Rashidi et al. (2015) analysed static and dynamic scheduling problem of AGVs for container terminal. Authors introduced graph based model algorithm called as Network Simplex Algorithm (NSA). It was observed that performance of algorithm found to be 100 times faster in comparison of simplex algorithm for solution of linear programming problems. Authors generated and fed random data to the model for approximately 50 AGVs. In the observations, NSA found to be fast and efficient in comparison of simplex algorithm. Erol et al. (2012) introduced a multi agent based approach for yield of feasible schedule using bidding criteria among the agents in real time manufacturing environment. Authors validated the method on offline problems from the literature. It was observed that generated real time schedules were comparable with other optimization algorithms. Fazlollahtabar et al. (2015) addressed multiple load AGVs scheduling issues for a manufacturing system. Authors found earliness and tardiness one of the significant factor for the satisfaction of cycle time of the process .Initially they proposed an analytical program for minimization of penalize earliness and tardiness later on a two stage optimization method was introduced for searching the solution space and optimal space.

Umar et al. (2015) applied integrated hybrid genetic algorithm and optimized various performance parameters of AGVs namely make span, AGV travel time, and penalty cost due to jobs tardiness and delay as a result of conflict avoidance. They performed integration of dispatch rules, scheduling rules, conflict-free routing of jobs and AGVs in the FMS facility. In algorithm a multi objective fitness function was applied and weights were assigned to each on the basis of performance improvement. Further authors applied fuzzy logic to control overall performance of algorithm. It was found that integrated scheduling of jobs with AGVs scheduling, dispatching and routing in FMS yield optimum results. A multi-load AGV can pick and drop additional loads simultaneously while processing previous load transfer request and considerably reduce trip time and total travel distance of empty AGVs.The performance of multi load AGVs was compared with unit load AGVs by Ulusoy et al. (1997). In simulation test it was observed that application of multi-load AGVs can increase throughput of the material handling system, considerably. Van der Meer et al. (2000) observed that a multi-load AGVs improves the system efficiency when multiple loads are picked from one particular location. It was also observed that with the use of multi load AGVs the scheduling of AGVs become complex and difficult to handle. Ho and Liao (2009) analyzed performance parameters such as through put and tardiness for multi load AGV systems operating under various load selection rules and performed simulation tests and found that pickup-dispatching rules and load-selection rules affect each other's performance. In simulation, it was also observed that the load-selection rule having common destination for load transfer yields best throughput and tardiness and the pickup-dispatching rule that dispatches AGV to the work center have highest loads in queue yields the best throughput and tardiness performance. Sadaghiani et al. (2014) applied Pareto archive floating search integrated heuristic for simultaneous optimization of three factor namely total workload, minimum make span and maximum workload of jobs. The aforesaid problem was divided into two parts considering assigning and sequencing sub problem. 
From literature review a research gap for scheduling of multi load AGVs for minimum travel and waiting time is observed. In present paper scheduling of multi load AGVs for minimum travel and waiting time is proposed by application of a novel modified memetic particle swarm optimization algorithm (MMPSO) and initial solutions developed by MMPSO and PSO (random and deterministic) are compared.

\section{Problem Definition}

\subsection{Assumptions and limitations}

The assumptions considered are as follows.

1. The path between two points is not necessarily unique and the system controller may change the route of AGVs to designated points due to congestion in the next lane or junction.

2. The number of work centers and their locations do not change until all jobs under consideration are completed.

3. The AGVs will not suffer from delays in the buffer area due to waiting for jobs.

4. Fleet of AGV is, $\mathrm{V}=\{1,2 \ldots,|\mathrm{V}|\}$ AGVs. Every AGV transports a few jobs. At the start of the process, the AGVs are assumed to be empty.

5. All AGVs are reliable and travel at predetermined constant average speed.

6. There is no job pre-emption.

7. Loading and unloading time of jobs on machine is included in the job's processing time.

8. Setup time on work center for all operations is assumed to be zero.

\subsection{Problem Statement}

In this scheduling problem, the aim is to deploy the AGVs in such a way that all the imposed constraints are satisfied and an optimized schedule with minimum travel and waiting time may be achieved. In this problem, the AGV can be at different locations in the FMS facility. It may be available at the work center, or in the middle of the guide path between its source and destination.

Let, $n=$ number of jobs.

Node $i$ and node $n+i=$ the pickup and delivery location of $i^{\text {th }}$ job in the network respectively In the network, different nodes can represent the same physical location of work center.

By adding node 0 and node $2 \mathrm{n}+1$, as the $\mathrm{AGV}$ initial start point and end point, to the network, the node set becomes as

$\mathrm{N}=\{0,1,2 \ldots, \mathrm{n}, \mathrm{n}+1, \mathrm{n}+2 \ldots, 2 \mathrm{n}, 2 \mathrm{n}+1\}$.

The pickup and delivery points are, respectively, included into two sets as

$\mathrm{P}^{+}=\{1,2 \ldots, \mathrm{n}\}$

$\mathrm{P}^{-}=\{\mathrm{n}+1, \mathrm{n}+2 \ldots, 2 \mathrm{n}\}$.

$\mathrm{P}=\mathrm{P}^{+} \cup \mathrm{P}^{-}$

$\mathrm{P}=$ Set of nodes for pickup and delivery points other than the FMS facility.

The following parameters are known:

a, Index for jobs; $\mathrm{a}=1,2, \ldots, \mathrm{m}$

$\mathrm{v}$, Index for AGVs; $\mathrm{v}=1,2, \ldots, \mathrm{n}$

$\mathrm{k}, \mathrm{k}$ ' Index for work center; $\mathrm{k}=1,2, \ldots, \mathrm{o}, \mathrm{k}^{\prime}=2,3, \ldots, \mathrm{o}$

$\mathrm{T}_{\mathrm{a}}=$ Appointment time of the $\mathrm{a}^{\text {th }}$ job.

$\mathrm{T}_{\mathrm{vo}}=$ Time at which the AGV, $\mathrm{v}$ leaves the work center.

$\mathrm{Q}_{\mathrm{v}}=$ Capacity of AGV, $\mathrm{v}$.

$\mathrm{s}=$ Number of jobs at work center

$p_{a k}=$ processing time of job "a" being processed at work center " $k$ "

$\mathrm{d}_{\mathrm{u}}=$ due date of job "a" being processed at work center " $k$ " 
$\mathrm{C}_{\mathrm{ak}}=$ completion time for job " $\mathrm{a}$ " being processed at work center " $\mathrm{k}$ "

$\mathrm{T}_{\mathrm{ij}}=$ Travel time from the physical location of node $\mathrm{i}, \mathrm{L}_{\mathrm{i}}$, to physical location of node $\mathrm{j}, \mathrm{Lj}$ (for Each pair of $i$ and $j$ in $\mathrm{N}$ ).

$X_{i j v}=$ Movement of AGV, "v" from node " $i$ " to node " $j "$ ".

If, $X_{i j v}=1$; $A G V$ " $v$ " moves from node "i" to node " $\mathrm{j}$ "; else $X_{i j v}=1$. So, its domain is $\{0,1\}$.

$\mathrm{Y}_{\mathrm{vi}}=\mathrm{Load}$ at AGV "v" when it leaves node "i".

Initially $\mathrm{Y}_{\mathrm{v} 0}=0$

$\mathrm{T}_{\mathrm{vi}}=$ Time at which the AGV v starts service at node " $\mathrm{i}$ "

Initially $\mathrm{T}_{\mathrm{v} 0}=0$

$\alpha ; \beta ; \delta ;=$ weights applied on the objective function due to loss of time.

\subsection{Objective Function and Constraints}

The constraints and objective function of the problem are formulated from Eq. (1) to Eq. 6. Equations represent the load of an AGV when it leaves the first pickup point after the work center and when AGV goes to any pickup or drop-off point after the first pickup. If a AGV goes to any pickup or drop-off point, its load will be increased or decreased by 1 hence AGV in material handling system will operate with multi loads after passing first pickup or drop- off point. The scheduling problem for multi load AGVs formulated in this paper is NP-hard problem and problem is formulated on the basis of constraint satisfaction and their optimization considering minimum travel and waiting time model. Configuration of multi load AGVs and work center is shown in Fig. 1.

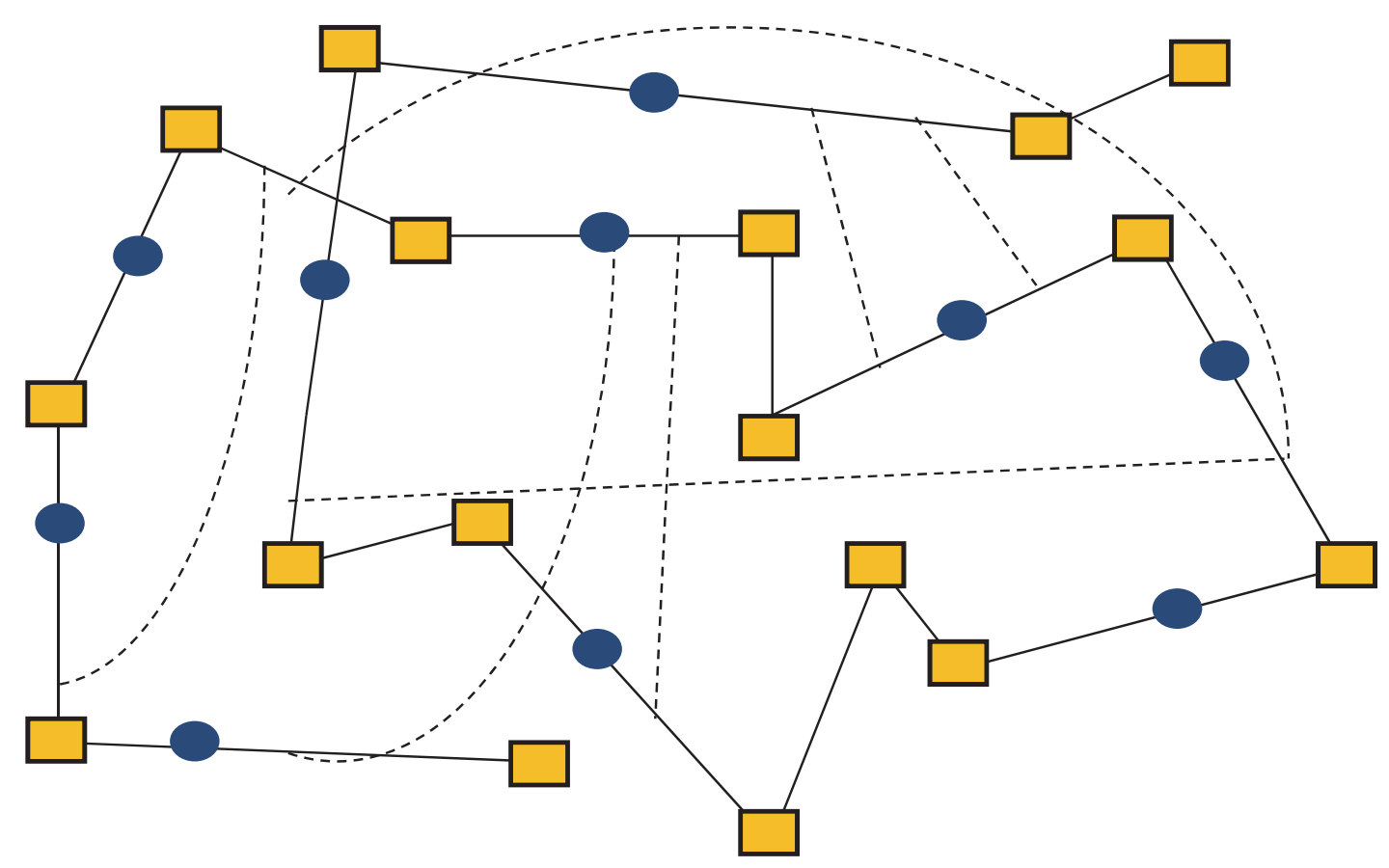

Multi Load AGV $\square$ Work Centre

Guide Path

Fig. 1. Configuration of AGVs and work centers 


$$
\begin{aligned}
& \text { If }\left(\mathrm{X}_{o j v}=1\right) \Rightarrow \mathrm{Y}_{\mathrm{vj}}=1 ; \mathrm{v} \in \mathrm{V}, \mathrm{j} \in \mathrm{P}^{+} \\
& \text {If }\left(\mathrm{X}_{\mathrm{ijv}}=1\right) \Rightarrow\left\{\begin{array}{c}
\mathrm{Y}_{\mathrm{vj}}=\mathrm{Y}_{\mathrm{vi}}+1 ; \mathrm{v} \in \mathrm{V}, \mathrm{j} \in \mathrm{P}^{+}, i \in P, i \neq \mathrm{j} \\
\mathrm{Y}_{\mathrm{vj}}=\mathrm{Y}_{\mathrm{vi}}-1 ; \mathrm{v} \in \mathrm{V}, \mathrm{j} \in \mathrm{P}^{-}, i \in P, i \neq \mathrm{j}
\end{array}\right\} \\
& \text { If }\left(\mathrm{X}_{0 j v}=1\right) \Rightarrow \mathrm{T}_{\mathrm{vj}}=\mathrm{T}_{\mathrm{v} 0}+\mathrm{T}_{\mathrm{L} 0, \mathrm{Lj}} ; \mathrm{j} \in \mathrm{P}^{+}, v \in V \text {, } \\
& \text { If }\left(\mathrm{X}_{i j v}=1\right) \Rightarrow \mathrm{T}_{\mathrm{vj}}=\mathrm{T}_{\mathrm{vi}}+\mathrm{T}_{\mathrm{Li}, \mathrm{Lj}} ; \mathrm{i}, \mathrm{j} \in \mathrm{P}, v \in V \text {, } \\
& \text { If }\left(\mathrm{X}_{i(2 n+1) v}=1\right) \Rightarrow \mathrm{T}_{\mathrm{v}(2 \mathrm{n}+1)}=\mathrm{T}_{\mathrm{vi}}+\mathrm{T}_{\mathrm{Li}, \mathrm{L}(2 \mathrm{n}+1)} ; \mathrm{i} \in \mathrm{P}^{-}, v \in V \\
& \sum_{v \in V} \sum_{j \in N} X_{\mathrm{ijv}}=1, \mathrm{i} \in \mathrm{P}^{+} \\
& \sum_{\mathrm{j} \in \mathrm{N}} \mathrm{X}_{\mathrm{ijv}}-\sum_{\mathrm{j} \in \mathrm{N}} \mathrm{X}_{\mathrm{jiv}}=1, i \in P, v \in V \\
& \sum_{\mathrm{j} \in \mathrm{N}} \mathrm{X}_{\mathrm{ijv}}-\sum_{\mathrm{j} \in \mathrm{N}} X j(n+i) v=1, i \in \mathrm{P}^{+}, v \in V \\
& \sum_{j \in P^{+}} X_{0 \mathrm{jv}}=1, \mathrm{v} \in \mathrm{V} \\
& \sum_{j \in P^{-}} X_{i(2 n+1)}=1, \mathrm{v} \in \mathrm{V} \\
& \mathrm{Y}_{\mathrm{vi}} \leq \mathrm{Q}_{\mathrm{v}}, \mathrm{v} \in \mathrm{V}, \mathrm{i} \in \mathrm{P} \\
& \min \text { time }=\sum_{\mathrm{v} \in \mathrm{V}}\left\{\alpha \sum_{i \in P} \sum_{j \in P, j \neq 1} \mathrm{X}_{\mathrm{ijv}} \cdot \mathrm{T}_{\mathrm{ij}}+\sum_{\mathrm{s}}\left(\beta \sum_{i \in P}\left|\mathrm{~T}_{\mathrm{i}}-\mathrm{T}_{\mathrm{vi}}\right|^{+}+\delta \sum_{i \in P}\left|T_{v i}-T_{i}\right|^{+}\right)\right\} \\
& \alpha=\sum_{k=1} \sum_{a=1}\left|p_{a k}-d u_{a k}\right|^{2} \\
& \beta=\sum_{k=1} \sum_{a=1}\left|p_{a k}-C_{a k}\right|^{2} \\
& \delta=\sum_{k=1} \sum_{a=1}\left|p_{a k}-q_{\mathrm{ak}}\right|^{2}
\end{aligned}
$$

Initially AGV leaves the work center and then it follows a pickup point. AGV can go to any pickup or delivery point after the first pickup. A multi load AGV before delivery to last work center delivers the job to second last work center. To calculate the starting service time at each node, the service time of the current node and the traveling time between the previous and current nodes should be considered. Constraints set on pickup and delivery points of the AGVs are applied. The constraint ensures that each pickup point is visited once by one of the AGV, if an AGV enters a node, it will exit the node and if an AGV visits a pickup node, then it has to visit the associated delivery node also.

The first visit of every AGV is a pickup node and the last visit of the AGV is a delivery node. The load of AGV "v" when it leaves node " $i$ " must not exceed the capacity of the AGV. Sum of traveling time of the AGVs to be calculated considering waiting rime of AGV and the lateness time to serve the jobs. If value of waiting time and lateness time of $A G V$ is positive then it will impact the objective function. 


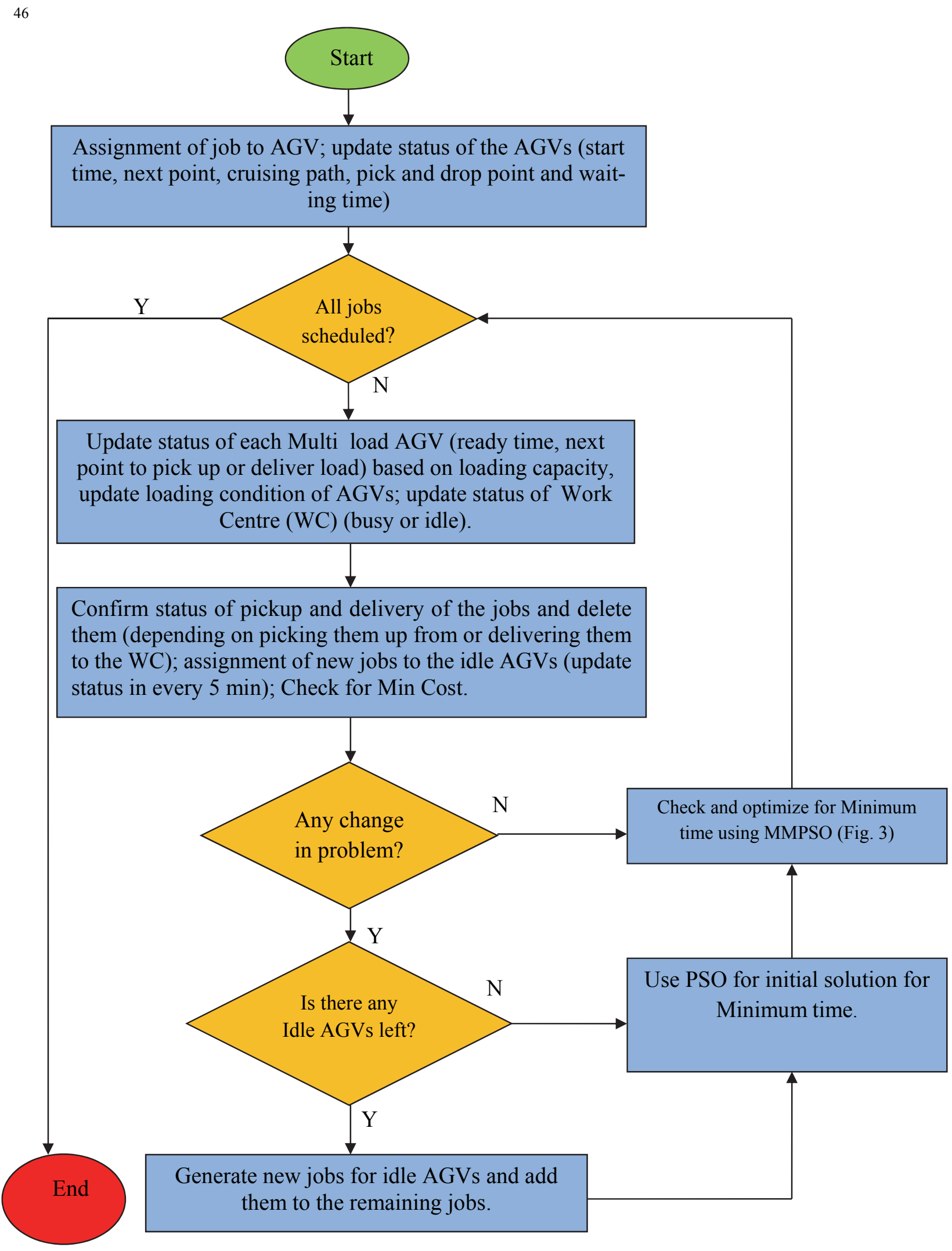

Fig. 2. Flow Chart for the process 


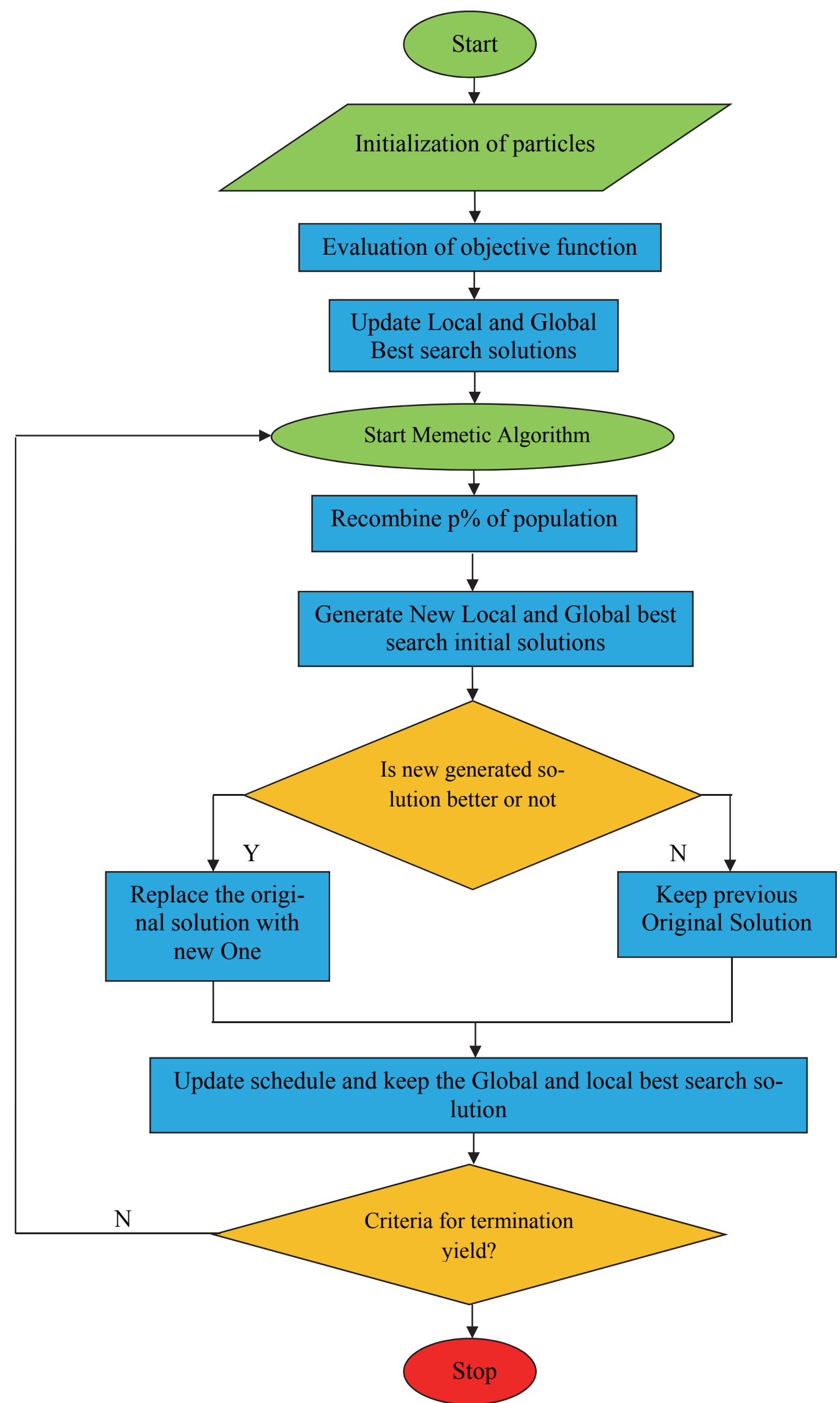

Fig. 3. Flowchart of MMPSO 


\section{Algorithms Overview}

\subsection{Particle Swarm Optimization Algorithm}

The objective of a PSO algorithm is to locate optimum area in a multi dimensional complex search space by interacting with other individual particle in population of particles. The particles are considered as moving points in the multi-dimensional space. The PSO algorithm is based on social interaction and search in space by dynamic movement of individual particle. Initially each particle has some initial velocity, position and advancement of each particle is based on particle velocity, which is also known as the best global position of the particle in the problem space (Kumar \& Sridharan, 2010).

In application of PSO algorithm the initialization of particle's population is carried out with random positions $\mathrm{p}(t)$ and velocities $\mathrm{v} i(t)$, for which the fitness functions, is evaluated. The position and the velocity for every particle changes at every iteration and every time the fitness function is evaluated in comparison to the new position and new velocity. Each iteration is carried out using Eq. (16)

The particle's position pi $(\mathrm{t})$, is compared with its previous generated solutions and if the new solution is better solution than the previous selected one, the new solution is stored as particle best position as $\mathrm{pi}^{\text {best }}$. The global best position (pgbest) which is best in the whole population is also stored. Particles change their velocities according to cognitive and social learning. Eq. (16) describes process of updating velocities of the particles.

$$
v_{i}(t+1)=v_{i}(t)+\left(c_{1} \times \text { rand }() \times\left(p_{i}^{\text {best }}-p_{i}(t)\right)\right)+\left(c_{2} \times \text { rand }() \times\left(p_{\text {gbest }}-p_{i}(t)\right)\right)
$$

where,

$\mathrm{Vi}(\mathrm{t}+1)$ new velocity for the $\mathrm{i}^{\text {th }}$ particle,

$\mathrm{c} 1$ and $\mathrm{c} 2$ weighting coefficients for the personal best and global best positions respectively,

$\mathrm{pi}(\mathrm{t}) \quad \mathrm{i}^{\text {th }}$ particle's position at time $\mathrm{t}$,

$\mathrm{pi}$ best $\quad \mathrm{i}^{\text {th }}$ particle's best known position,

pgbest best position known to the swarm.

The rand ( ) function generate a uniformly random variable $\in[0,1]$.

Eq. (16) updates the position and velocity of individual particle and considers the best positions within a particles local neighborhood at time interval $t$.

A particle's position is updated using Eq. (17).

$$
p_{i}(t+1)=p_{i}(t)+v_{i}(t)
$$

First part of the Eq. (16) represents the velocity component of the previous iteration. The second part is known as cognitive component which keeps on interacting between the particle's current position and its best position. The interaction phase of the particles is also known as social learning .Particle's new position is updated by Eq. (17). Pseudo code for Particle Swarm Optimization Algorithm for Minimum Cost is mentioned below in Fig. 4. 


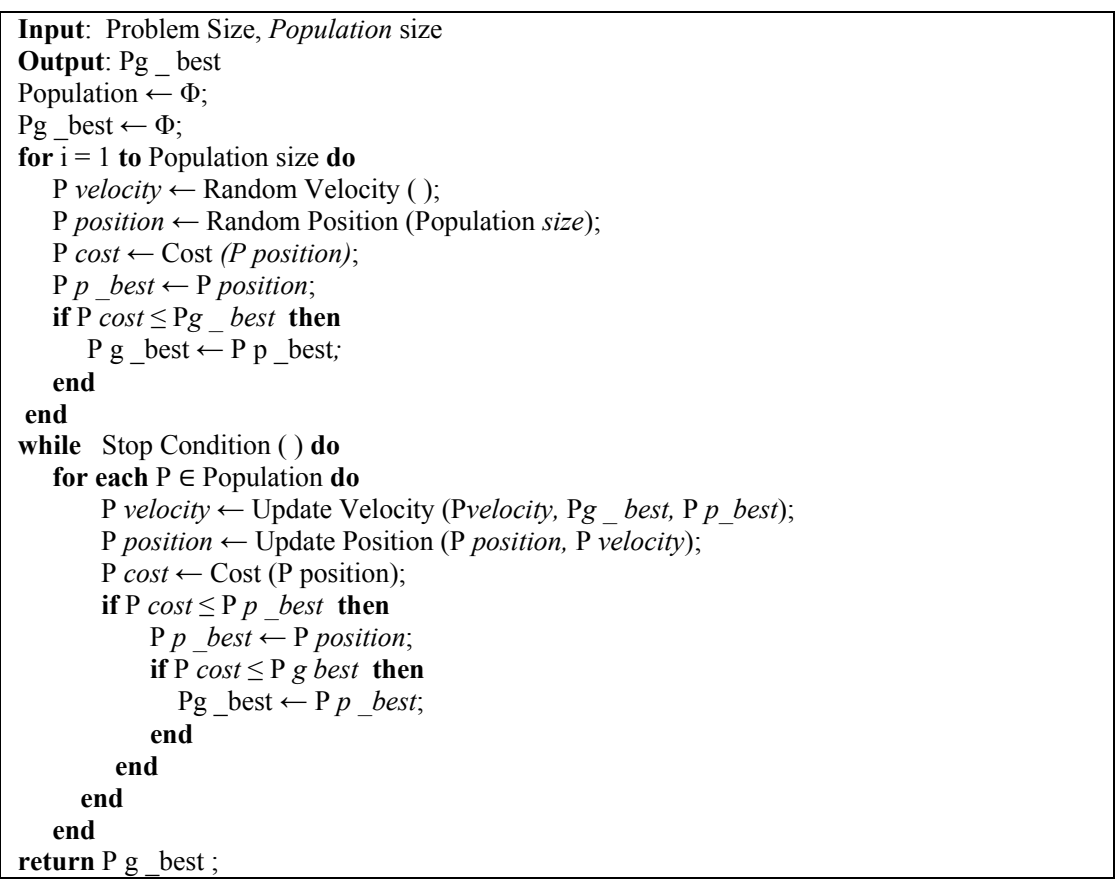

Fig. 4. Pseudo code for Particle Swarm Optimization Algorithm for Minimum Cost

\subsection{Memetic Algorithm}

Memetic Algorithms are developed after applying interplay of evolution and genetic evolution. The algorithm implies the generalization of genes to discrete systems in which information is inherited and the discrete systems is subjected to some evolutionary forces of selection and variation. The term 'meme' is generally applied to a unit of discrete system's cultural information, indicating about the interplay of genetic and cultural evolution together. The information processing process exploits the population by applying global search technique and locates good population areas in the search space. Further the method is used in combination of repeated application of local search heuristic by individual solutions and locates the local optimum. The Memetic Algorithm applies canonical Genetic Algorithm as the global search method which functions on binary strings, applies tournament selection, point mutations, uniform crossover and a binary coded decimal decoding of bits to real values. Local search performs probabilistic bit flipping (point mutations) and selects solution with same or improved fitness. The memetic algorithms exhibit the dual capabilities of genetic evolution as well as cultural evolution which perform process including transmission, selection, inheritance, and variation of memes as well as genes. Pseudo code for Memetic Algorithm is mentioned below in Fig. 5.

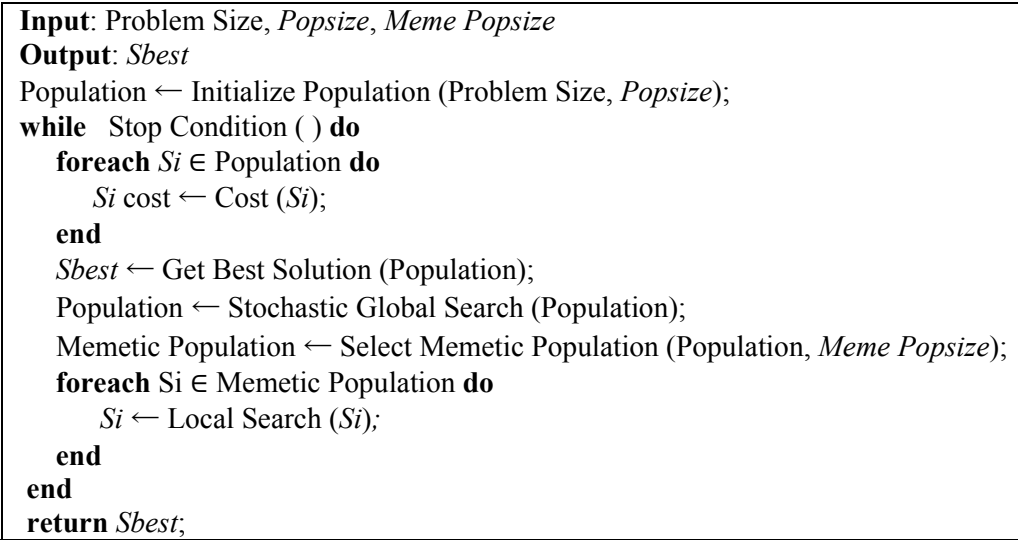

Fig. 5. Pseudocode for Memetic Algorithm 
To present neighborhood solution for the MA three options of job subsequences for each multi load AGV can be applied as

i) Sub sequence of job relocation for each multi load AGV - Insert a job or a sequence of jobs served by AGV from one route into another route.

ii) Sub sequence of job exchange for each multi load AGV-Improve the solution for the AGV routing problem by exchanging jobs or subsequence of jobs between two routes.

iii) Sub sequence of job mix for each multi load AGV - combined above two methods and tries to relocate, exchange jobs or subsequence of jobs, and simultaneously compute the alternatives to find the alternative generating the minimum travel and waiting time.

In this paper we considered the subsequence of job mix for each $\mathrm{AGV}$, which yield better solution.

\subsection{Modified Memetic Particle Swarm Optimization Algorithm (MMPSO) for Multi Load AGVS}

Algorithm's performance is largely dependent on its capability of exploration of solutions (global search) and exploitation of solutions (local search). In Particle swarm optimization algorithm (PSO), particles under consideration converge towards specific points prematurely at early stage as the particles get attracted to the global best position and hence a little consideration is given to exploitation process in comparison to exploration process. The exploitation process for good local search by an algorithm can be improved by applying evolutionary algorithms which proved to be very useful in many applications. It is necessary to improve local search capabilities in optimization so that optimum solutions could be generated. In this paper the memetic algorithm (MA), for optimum local solution and particle swarm optimization (PSO) algorithm for optimum global solution is integrated and applied for multi load AGVs minimum travel and waiting time scheduling in FMS. The new algorithm is named as modified memetic particle swarm optimization (MMPSO). The MMPSO improve the local search ability by combining the solutions (particle's position) similar to the use of crossover in GAs. The recombination process is applied to random selected $\mathrm{p} \%$ of the population of the solutions. After recombining solutions the solutions generally yields better fitness values in comparison to original or previous solution values and hence new solutions are replaced with previous solutions. The new recombined solutions generated from sufficient local search bring balance between exploration process and exploitation process of the algorithm and hence the algorithm yields good solutions for minimum travel and waiting time as shown in figure of flowchart 2 and figure of flowchart 3.Particle Swarm Optimization (PSO) and Memetic Algorithm (MA) combined together can generate optimum solutions for Multi load AGVs scheduling problem for minimum travel and waiting time as shown in Fig. 6.

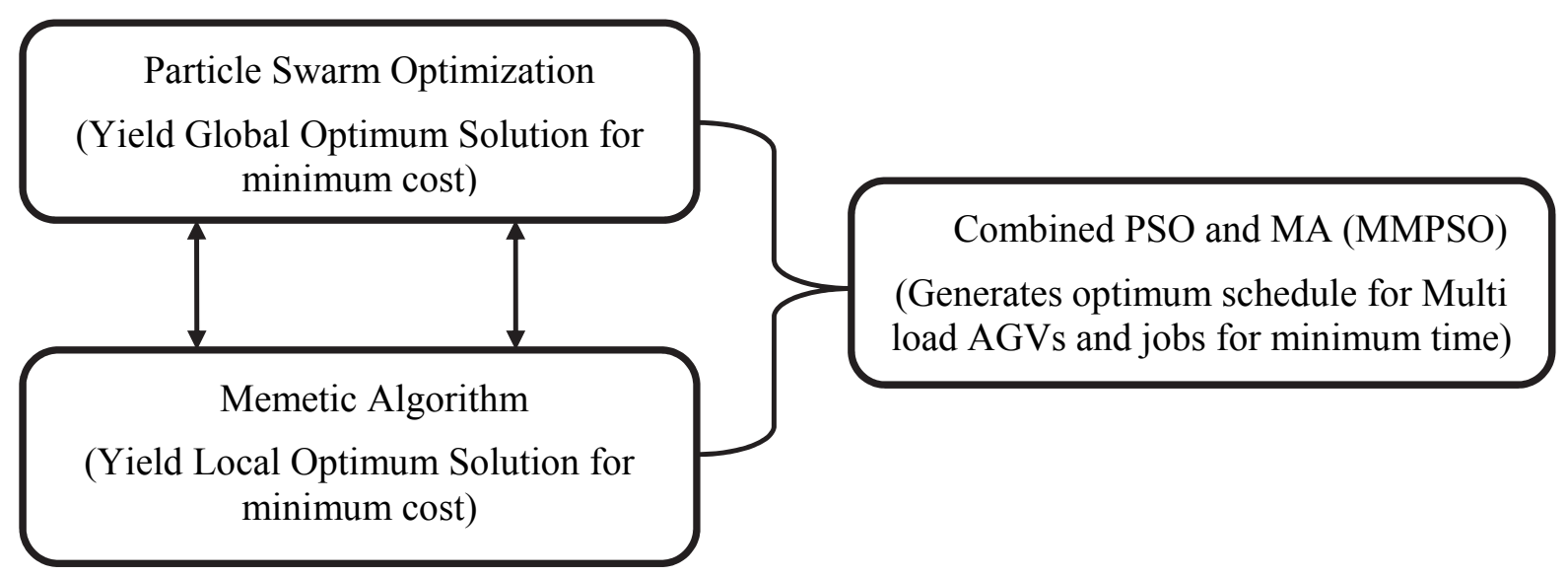

Fig. 6. Integrated PSO and MA as MMPSO

The following three methods are considered to get an initial feasible solution for the PSO and MMPSO: 
i. Deterministic initial feasible solution by PSO: In this case, the travel distance for each AGV equals to the total number of jobs divided by the total number of AGVs.

ii. Random initial feasible solution by PSO: Some random jobs are chosen so satisfy the feasibility constraints. This approach allows the process to begin at different neighborhoods.

iii. Solutions from MMPSO: The initial optimal solution from PSO is generated for single-loaded condition for AGVs after getting initial solution; MMPSO is applied again to find a better solution for multi load AGVs.

The application of integrated PSO and MA as MMPSO is based on the third method for the initial feasible optimum solution as shown in flowcharts of figure 2 and 3.

\section{Experimental Results}

The proposed model and algorithms were tested on a hypothetically designed manufacturing facility. The program was run in $\mathrm{C}++$ to perform test on some randomly generated problems .The generated results and data for problems of $20 \mathrm{AGVs}$ and 35 work centers is discussed. The combined PSO and MA (MMPSO) algorithm was applied for multi load AGVs problem. The model was solved by conducting number of iterations in MMPSO and same are mentioned in Table 1.

\section{Table 1}

Comparison of Initial Feasible Solutions by PSO and MMPSO

\begin{tabular}{|c|c|c|c|c|c|c|c|}
\hline \multirow[b]{3}{*}{$\begin{array}{l}\text { Problem } \\
\text { No. }\end{array}$} & \multirow[b]{3}{*}{ Jobs } & \multirow[b]{3}{*}{$\begin{array}{c}\text { Iterations } \\
\text { by MMPSO }\end{array}$} & \multirow{3}{*}{$\begin{array}{l}\text { Colum (A) - } \\
\text { Initial Feasible } \\
\text { Solution } \\
\text { (MMPSO) }\end{array}$} & \multicolumn{4}{|c|}{ Objective function value by PSO for multi load AGVs and their Differences } \\
\hline & & & & \multicolumn{2}{|c|}{ Initial Feasible Solution (Deterministic) } & \multicolumn{2}{|c|}{ Initial Feasible Solution (Random) } \\
\hline & & & & $\begin{array}{l}\text { Objective Func- } \\
\text { tion }\end{array}$ & $\begin{array}{l}\text { Percentage In- } \\
\text { crease, Compari- } \\
\text { son with Colum } \\
\text { (A) }\end{array}$ & $\begin{array}{l}\text { Objective } \\
\text { Function }\end{array}$ & $\begin{array}{l}\text { Percentage In- } \\
\text { crease, Compari- } \\
\text { son with Colum } \\
\text { (A) }\end{array}$ \\
\hline 1 & 10 & 97 & 2,356 & 4,254 & 44.62 & 4,586 & 48.63 \\
\hline 2 & 15 & 118 & 5,121 & 8,545 & 40.07 & 8,985 & 43.01 \\
\hline 3 & 20 & 155 & 7,256 & 10,521 & 31.03 & 10,456 & 30.60 \\
\hline 4 & 25 & 165 & 10,005 & 14,526 & 31.12 & 15,487 & 35.40 \\
\hline 5 & 30 & 223 & 14,258 & 18,765 & 24.02 & 19,745 & 27.79 \\
\hline 6 & 35 & 285 & 20,148 & 26,452 & 23.83 & 28,456 & 29.20 \\
\hline 7 & 40 & 328 & 28,965 & 35,547 & 18.52 & 37,786 & 23.34 \\
\hline 8 & 45 & 333 & 35,659 & 40,258 & 11.42 & 42,879 & 16.84 \\
\hline 9 & 50 & 545 & 42,578 & 47,587 & 10.53 & 49,214 & 13.48 \\
\hline 10 & 55 & 456 & 48,756 & 54,241 & 10.11 & 56,214 & 13.27 \\
\hline 11 & 60 & 520 & 55,147 & 61,156 & 9.83 & 62,248 & 11.41 \\
\hline 12 & 65 & 620 & 64,287 & 70,431 & 8.72 & 71,579 & 10.19 \\
\hline 13 & 70 & 671 & 72,459 & 78,521 & 7.72 & 80,253 & 9.71 \\
\hline 14 & 75 & 616 & 84,621 & 90,428 & 6.42 & 93,128 & 9.13 \\
\hline 15 & 80 & 856 & 92,186 & 98,052 & 5.98 & 101,253 & 8.95 \\
\hline 16 & 85 & 825 & 99,854 & 105,238 & 5.12 & 108,471 & 7.94 \\
\hline 17 & 90 & 1,019 & 107,259 & 112,984 & 5.07 & 116,276 & 7.75 \\
\hline 18 & 95 & 1,136 & 115,784 & 123,215 & 6.03 & 127,547 & 9.22 \\
\hline 19 & 100 & 1,325 & 121,478 & 133,176 & 8.78 & 137,429 & 11.61 \\
\hline 20 & 110 & 1,533 & 136,457 & 151,547 & 9.96 & 156,254 & 12.67 \\
\hline 21 & 120 & 1,657 & 153,248 & 171,257 & 10.52 & 178,695 & 14.24 \\
\hline 22 & 130 & 1,945 & 176,842 & 199,243 & 11.24 & 210,549 & 16.01 \\
\hline 23 & 140 & 2,154 & 195,249 & 230,437 & 15.27 & 240,869 & 18.94 \\
\hline 24 & 150 & 2,426 & 235,496 & 288,621 & 18.41 & 299,519 & 21.38 \\
\hline 25 & 160 & 2,675 & 293,179 & 365,159 & 19.71 & 387,128 & 24.27 \\
\hline
\end{tabular}

In Table 1 the objective function for multi load AGVs which represents waiting times, traveling times of AGVs and lateness time to serve jobs in the manufacturing facility as initial feasible solution were calculated by PSO method and same are compared with initial feasible solution generated by MMPSO mentioned in column (A). The percentages of increase in the values of initial feasible solution by PSO method (Deterministic and Random) and values obtained by MMPSO method was also calculated and compared in the Table 1. Further the results are presented in form of graph in Fig. 7. Values of initial feasible solutions mentioned in Table 1, Deterministic and Random are analyzed statistically using 
student's t-test with $5 \%$ of rejection on the true hypothesis considering two equal means and the results of the aforesaid analysis are represented in Table 2 and Table 3 in form of values of t-test (Paired two sample for means), t-critical two-tail (Distribution) and Pearson Correlation for a particular degree of freedom. The student's t-test yields that the means are significantly different at a $95 \%$ degree of confidence as mentioned in Table 3 and Table 4. From the results obtained and mentioned in Table 1, it is crystal clear that the objective function, (initial feasible solutions) generated by PSO (deterministic initial feasible solution and random initial feasible solution) is approximately $48 \%$ and $7 \%$ deteriorated, respectively, as compared with the objective function (initial feasible solution) generated by MMPSO compared to results of multi load AGVs mentioned in Colum 6 and 8 as percentage increase and comparison with Colum (A). The PSO directly reaches to the global solution for the problem and then the MMPSO continues to find and yield more optimal local solution for the problem. Hence, MMPSO yields significantly good initial feasible solutions for the problem.

Table 2

Mean and Variance of Initial Feasible Solution alternatives PSO and MMPSO (Deterministic; Random)

\begin{tabular}{ccccc}
\hline S No. & $\begin{array}{c}\text { Statistical Parame- } \\
\text { ters }\end{array}$ & $\begin{array}{c}\text { Initial Feasible So- } \\
\text { lution (MMPSO) }\end{array}$ & $\begin{array}{c}\text { Initial Feasible Solu- } \\
\text { tion -Deterministic } \\
\text { (PSO) }\end{array}$ & $\begin{array}{c}\text { Initial Feasible So- } \\
\text { lution -Random } \\
\text { (PSO) }\end{array}$ \\
\hline 1 & Mean & $88,745.92$ & $1,01,606.44$ & $1,05,800.24$ \\
2 & Variance & $58,68,034,600.99$ & $84,42,174,879.09$ & $93,22,388,682.35$ \\
3 & Observations & 25 & 25 & 25 \\
\hline
\end{tabular}

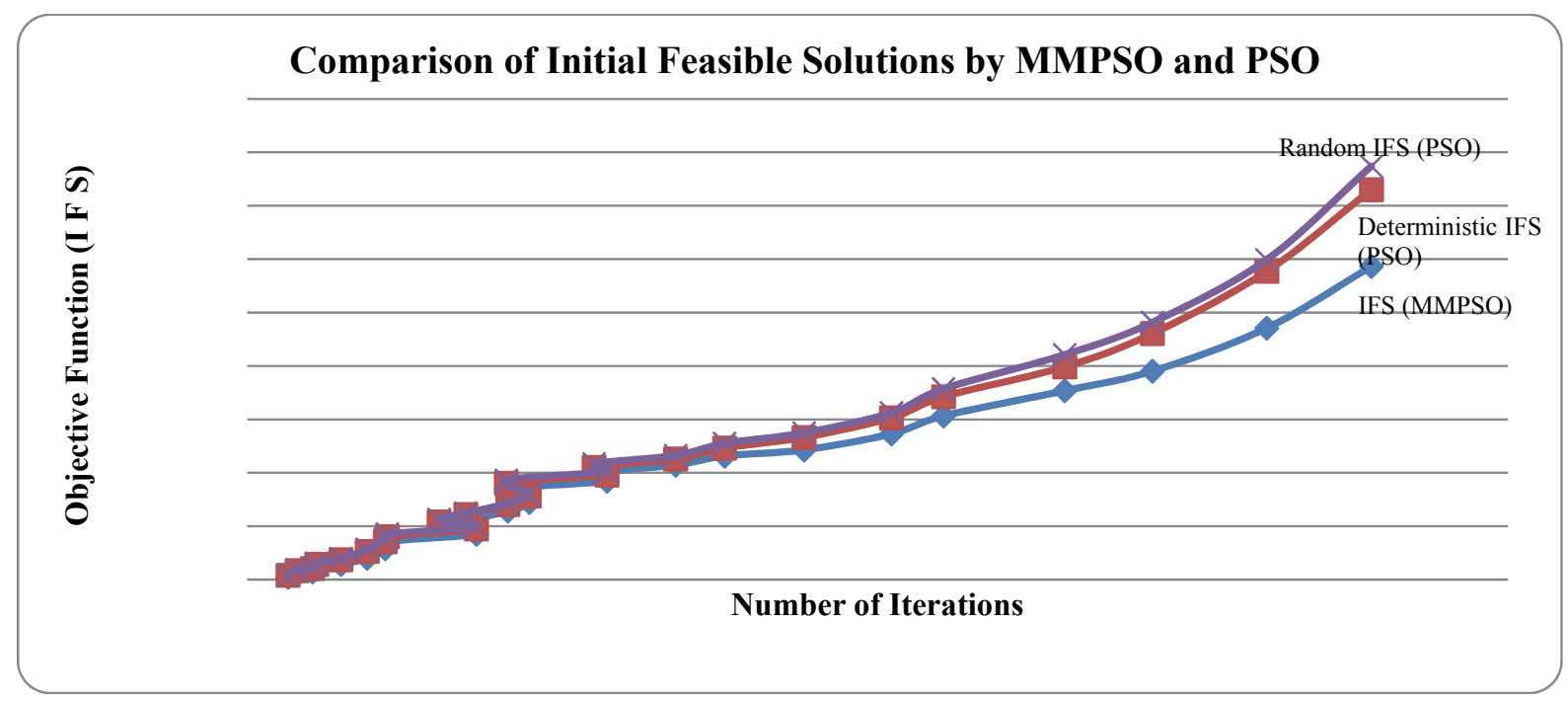

Fig. 7. Comparison of Initial Feasible Solutions by MMPSO and PSO

Table 3

Student's t-Test Result (Paired two sample for means) between Initial Feasible Solution alternatives PSO and MMPSO (Deterministic; Random)

\begin{tabular}{lccc}
\hline S No. & Statistical Parameters & $\begin{array}{c}\text { Initial Solutions (MMPSO) and } \\
\text { Initial Solution (Deterministic- } \\
\text { PSO) }\end{array}$ & $\begin{array}{c}\text { Initial Solutions (MMPSO) and Ini- } \\
\text { tial Solution (Random-PSO) }\end{array}$ \\
\hline 1 & Observations & 25 & 25 \\
2 & t-test ( Paired two sample for means) & -3.81473842786227 & -3.95311173533888 \\
3 & Degree of freedom & 24 & 24 \\
4 & t-Critical two-tail (Distribution) & 2.06389854731807 & 2.06389854731807 \\
5 & Pearson Correlation & 0.996397394210632 & 0.995449158629143 \\
6 & $\mathrm{P}(\mathrm{T}<=\mathrm{t})$ two-tail & 0.000840359754730043 & 0.00059313798741914 \\
\hline
\end{tabular}




\section{Conclusion and Future Work}

In the present paper, an integration of Particle Swarm Optimization Algorithm (PSO) and Memetic Algorithm (MA) as Modified Memetic Particle Swarm Optimization Algorithm (MMPSO) has been applied for finding optimum initial solutions (waiting times, traveling times of AGVs and lateness time to serve jobs in the manufacturing facility) for scheduling of multi load AGVs serving in FMS and their results were compared with initial solutions of scheduling of AGVs by PSO Algorithm. Although there have been some studies for the scheduling of unit load AGVs and very few studies on multi load AGVs but our research yields initial solutions for scheduling of multi load AGVs with minimum travel and waiting time for real time material handling operations, which promise a new horizon for future research towards scheduling of multi load AGVs for minimum travel and waiting time and minimum cost by application of integrated evolutionary algorithms. The MMPSO applied for multi load AGVs can deliver significant throughput when apply in service to real time material handling activities in FMS. The proposed system has been explained in detail and validated with test problem. The results from the integrated application of PSO and MA as MMPSO conclude that the MMPSO can yield promising results for real-time dynamic scheduling of multi load AGVs in comparison to the PSO algorithm applied for multi load AGVs and hence performance of MMPSO found to be very effective and much better than that of the PSO. In computational result the proposed method found to be efficient and better in generation of optimum initial solutions in comparison to other methods for the scheduling problems of multi load AGVs.

\section{References}

Akturk, M. S., \& Yilmaz, H. (1996). Scheduling of automated guided vehicles in a decision making hierarchy. International Journal of Production Research, 34(2), 577-591.

Egbelu, P. J., \& Tanchoco, J. M. A. (1986). Potentials for bi-directional guide-path for automated guided vehicle based systems. International Journal of Production Research, 24(5), 1075-1097.

Erol, R., Sahin, C., Baykasoglu, A., \& Kaplanoglu, V. (2012). A multi-agent based approach to dynamic scheduling of machines and automated guided vehicles in manufacturing systems. Applied soft computing, 12(6), 1720-1732.

Fazlollahtabar, H., Saidi-Mehrabad, M., \& Balakrishnan, J. (2015). Mathematical optimization for earliness/tardiness minimization in a multiple automated guided vehicle manufacturing system via integrated heuristic algorithms. Robotics and Autonomous Systems, 72, 131-138.

Fleischmann, B., Gnutzmann, S., \& Sandvoß, E. (2004). Dynamic vehicle routing based on online traffic information. Transportation science, 38(4), 420-433.

Gaskins, R. J., \& Tanchoco, J. M. (1987). Flow path design for automated guided vehicle systems. International Journal of Production Research, 25(5), 667-676.

Gaskins, R. J., Tanchoco, J. M. A., \& Taghaboni, F. (1989). Virtual flow paths for free-ranging automated guided vehicle systems. The International Journal of Production Research 27(1), 91-100.

Grunow, M., Günther, H. O., \& Lehmann, M. (2005). Dispatching multi-load AGVs in highly automated seaport container terminals. Container Terminals and Automated Transport Systems Part I, 231-255.

Ho, Y. C., \& Liao, T. W. (2009). Zone design and control for vehicle collision prevention and load balancing in a zone control AGV system. Computers \& Industrial Engineering, 56(1), 417-432.

Jerald, J., Asokan, P., Saravanan, R., \& Rani, A. D. C. (2006). Simultaneous scheduling of parts and automated guided vehicles in an FMS environment using adaptive genetic algorithm. The International Journal of Advanced Manufacturing Technology, 29(5), 584-589.

Kumar, N. S., \& Sridharan, R. (2010). Simulation-based metamodels for the analysis of scheduling decisions in a flexible manufacturing system operating in a tool-sharing environment. The International Journal of Advanced Manufacturing Technology, 51(1-4), 341-355. 
Levitin, G., \& Abezgaouz, R. (2003). Optimal routing of multiple-load AGV subject to LIFO loading constraints. Computers \& Operations Research, 30(3), 397-410.

Mantel, R. J., \& Landeweerd, H. R. (1995). Design and operational control of an AGV system. International Journal of Production Economics, 41(1-3), 257-266.

Meersmans, P. J. M. (2002). Optimization of container handling systems.

Nayyar, P., \& Khator, S. K. (1993). Operational control of multi-load vehicles in an automated guided vehicle system. Computers \& industrial engineering, 25(1-4), 503-506.

Powell, W. B., Towns, M. T., \& Marar, A. (2000). On the value of optimal myopic solutions for dynamic routing and scheduling problems in the presence of user noncompliance. Transportation Science, 34(1), 67-85.

Qiu, L., Hsu, W. J., Huang, S. Y., \& Wang, H. (2002). Scheduling and routing algorithms for AGVs: a survey. International Journal of Production Research, 40(3), 745-760.

Rashidi, H. (2010). Scheduling in container terminals using Network Simplex Algorithm. Journal of Optimization in Industrial Engineering, 9-16.

Rashidi, H., \& Tsang, E. (2015). Vehicle Scheduling in Port Automation: Advanced Algorithms for Minimum Cost Flow Problems. CRC Press.

Sadaghiani, J., Boroujerdi, S., Mirhabibi, M., \& Sadaghiani, P. (2014). A Pareto archive floating search procedure for solving multi-objective flexible job shop scheduling problem. Decision Science Letters, 3(2), 157-168.

Ulusoy, G., Sivrikaya-Șerifoğlu, F., \& Bilge, Ü. (1997). A genetic algorithm approach to the simultaneous scheduling of machines and automated guided vehicles. Computers \& Operations Research, 24(4), 335-351.

Umar, U. A., Ariffin, M. K. A., Ismail, N., \& Tang, S. H. (2015). Hybrid multiobjective genetic algorithms for integrated dynamic scheduling and routing of jobs and automated-guided vehicle (AGV) in flexible manufacturing systems (FMS) environment. The International Journal of Advanced Manufacturing Technology, 81(9-12), 2123-2141.

Van der Meer, R. (2000). Operational control of internal transport(No. TTS; T2000/5).

Veeravalli, B., Rajesh, G., \& Viswanadham, N. (2002). Design and analysis of optimal material distribution policies in flexible manufacturing systems using a single AGV. International journal of production research, 40(12), 2937-2954.

Yang, C., Choi, Y., \& Ha, T. (2004). Simulation-based performance evaluation of transport vehicles at automated container terminals. OR spectrum, 26(2), 149-170.

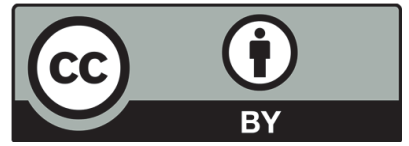

(C) 2018 by the authors; licensee Growing Science, Canada. This is an open access article distributed under the terms and conditions of the Creative Commons Attribution (CC-BY) license (http://creativecommons.org/licenses/by/4.0/). 